\title{
Automatic processing analysis of infrared images for monitoring pantograph catenary interactions
}

\author{
A. Balestrino, O. Bruno, A. Landi \& L. Sani \\ Dipartimento di Sistemi Elettrici e Automazione, Università di Pisa, Italy
}

\begin{abstract}
This paper shows a new sensor based on infrared images for monitoring pantograph catenary interaction. Due to the displacement of the contact point with respect to the reference position an image processing analysis of each frame is performed by using the Hough transformation in order to detect automatically monitoring variables of interest (e.g. temperature at the contact point and the position of the support towers along the railway line).

Keywords: infrared image, pantograph-catenary interaction, Hough transformation, segment detection.
\end{abstract}

\section{Introduction}

In order to improve the maintenance activities, a relevant objective for railway companies is the development of new sensors for a continuous monitoring of the quality of the current transmission between the overhead line and the collector strips of the pantograph. This problem is grown crucial with the introduction of the European Standard 96/48/CE about the interoperability. It is well-known that a poor electric contact between is the origin of arcing between the overhead wire and the collector strips of the pantograph. Several investigations have shown that the main damages of the overhead contact line installations are caused by the short term thermal effect of these arcing. The process which leads to the deterioration of the contact wire is associated with a localised recrystallization of the copper (i.e. a transaction to a stable crystalline microstructure with loss of all physical characteristics typical of the cold-drawn) and the formation of pits and dents on the surface. Unfortunately high speeds worsen the problem and a monitoring system has to be set up and tested to plan maintenance activity. 
Previous research activity (Bruno et al [1], Barmada et al [2]) revealed that a new measurement system, based on a phototube sensor, could detect break arcs between the pantograph and the contact line. A different approach is a measurement of the quality of the overhead contact by using infrared cameras. Preliminary results based on the analysis of infrared images were proposed by Balestrino et al [3]. Measurements carried out during high speed test runs and a post processing of the data collected have revealed the effectiveness of the proposed method for detecting the losses of contact, in order to evaluate and test the performance of running pantographs and of the contact wires. The task of the proposed research is the application of tracking algorithm of predefined objects (e.g., the contact point on the pantograph strip) based on the Hough transform over a sequence of images acquired from thermo-camera, in order to monitoring variables of interest, (e.g., temperature at the contact point and the position of the support towers along the railway line).

\section{Thermography for monitoring pantograph-catenary interactions}

Thermo-cameras have not been used extensively in railway research. Main advantages offered by thermo vision are:

a) it is a non-contact and non-destructive technique;

b) it is suited to monitoring devices operating under high voltage or carrying high currents;

c) is more informative than a standard camera image, because of its insensitivity to different weather conditions, to the daily-nightly runs or to the presence of tunnels.

A critical aspect of thermo vision is that the exact temperature of the body under test cannot be directly revealed. Nevertheless infrared imaging is an excellent method for extracting a qualitative map of the superficial temperature. As a matter of fact thermal analysis gives a relative information on the temperature, following the reasoning of Runciman [4]: all objects at temperatures above absolute zero emit electromagnetic radiation. Radiation thermometry makes use of this fact to estimate the temperatures of objects by measuring the radiated energy from selected regions.

Every physical process characterized by an increase or decrease in surface temperature is detectable with infrared thermography. The intensity of the emitted radiation depends on two factors: the body temperature and the factor emissivity of the surface, i.e. the ability of the object to radiate, defined by the Stefan-Boltzmann equation:

$$
E=\varepsilon \sigma T^{4}
$$

where: $\mathrm{E}$ is the radiation in $\mathrm{W} / \mathrm{m}^{2}, \varepsilon$ the emissivity, $\sigma$ the Boltzmann constant and $\mathrm{T}$ is the temperature in Kelvin. 
The emissivity factor $\varepsilon$ lies between zero and the unity, depending on the material nature and on the superficial roughness. Infrared imaging systems convert infrared heat emissions into a picture that shows the relative temperature differences in a range of grey tones, or in a series of colours, in such a way that the desired temperature information is easily interpreted by the user. In fig. 1.a a typical infrared image of pantograph-catenary interaction in the case of a burst of arcing (i.e. losses of contact between catenary and pantograph) is shown (the train speed was $200 \mathrm{~km} / \mathrm{h}$ in this case). The maximum infrared emission is in the neighbourhood of the contact surface between strip and wire. Interaction region between the overhead feeder and the strip shows a high thermal gradient. A continuous monitoring of the contact region leads to interesting information on the quality of the current transmission.

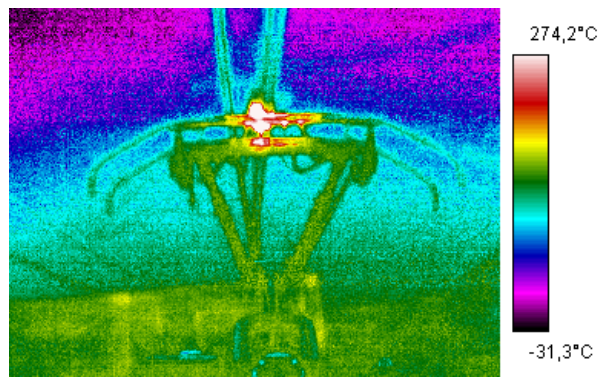

Figure 1: Thermal image in case of a burst of arcing.

\section{The automatic image processing}

It is well-known that the contact point between the pantograph strip and the overhead line is not fixed. In fact the wire is zigzagged relative to the centre line of the track, to even the wear on the train's pantograph as it runs underneath. Moreover, because of the no uniform elasticity of the catenary, the height of the contact wire may change (e.g. entering a tunnel). Therefore a relevant displacement of the contact point may complicate the image processing, unless considering the knowledge of the moving spatial coordinates of the contact area. A first step for an automatic image processing analysis is to follow frame-byframe the position of some critical features (pantograph strips, overhead electric line). All these elements can be characterized by linear contours. This observation allows the use of standard algorithms for detecting straight lines inside each image under examination.

The Hough transform is well-suited for detecting straight-lines in the real image: its application can be extremely useful for monitoring the contact point, characterized from the intersection of a segment representing the strip and a straight line representing the overhead line contact. Furthermore it can identify different rectilinear structures, such as the poles along the railway line, helping in finding a more precise correlation between the overheating of the contact point (or a burst of arcing) and the position of the train. A detailed description of the Hough transform is reported by Hough [5] and Deans [6]. 
The object recognizing procedure is depicted in the followings steps.

a) Independently from the electronic format of the recorded file (we considered outputs in .bmp, .mpg or .avi formats), single frames are extracted from the image sequence. Each image is first stored in a matrix, then converted from true colour (RGB) to grey scale.

b) Each image is suitably filtered for reducing noises and sharpen objects, then edges are extracted and image is then converted from a grey scale to a binary image. Edge detection was performed using Canny algorithm with a preliminary noise reduction (Canny [7]). After this step, each frame is represented by a binary image with edges evidenced. It is an easy task to identify the pantograph, the overhead contact lines and the poles.

c) Object locations in every frame is characterised from an analysis of edges and a detection of reference straight lines. All straight lines can be localised using the Hough transform.

In order to increase algorithm efficiency and to reduce computational burden, we suppose to know, within a limited variation, the position and the possible movement of the features to be monitored, therefore two observation windows into the image are locked: a first one includes the pantograph structure and the second one reveals the passage of the support structures. The straight line search can be limited inside these rectangular regions of the image.

The procedure allows a quick detection of the object position and a repetitive algorithm allows following the trajectory of an object inside successive frames. After a first detection of the trajectory, it is a good strategy to eliminate frames with discontinuous trends of the object and to filter the image for reducing noise and to sharpen the image.

Following this logic a software tool was created: it allows the user to detect automatically:

1. pantograph strip position;

2. catenary wires;

3. position of the poles.

This procedure can be repeated for each frame of the sequence. Since the pantograph moves slowly with respect the frame rate of the camera, we can use the position of the strip detected in the previous image to window next image appropriately. So the computation time can be greatly reduced.

\subsection{Position of the pantograph inside an image}

The pantograph appears at each frame and its position moves slowly. Small displacements both in vertical and in horizontal direction are allowed: they are due to the movements of the main frame and of the contact arms.

In thermo images are evidenced two different pantograph heads and two different strips: the goal of the following study is to follow only the strip nearest the IR camera. Contact analysis is applied to the squared window of fig. 2.a, named reference box: its size and position inside the image will remain unchanged for the limited displacement of the pantograph head. Several segments are easily recognised by Hough transform (fig. 2.a): only the upper 


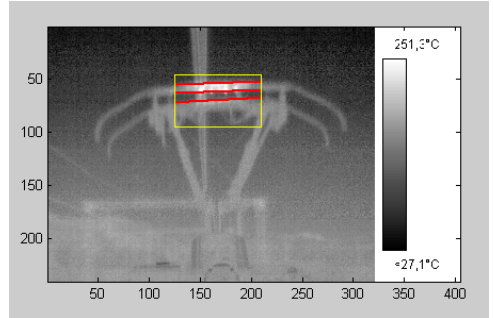

(a)

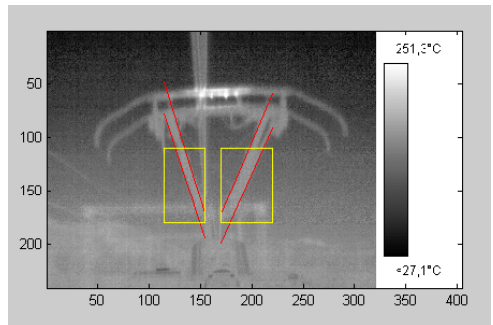

(c)

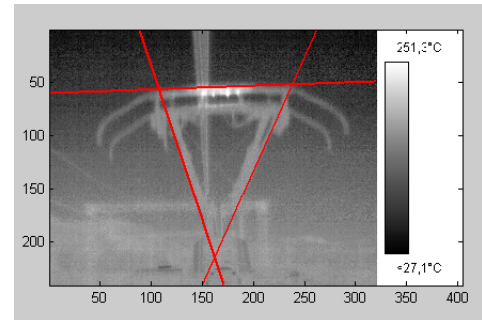

(e)

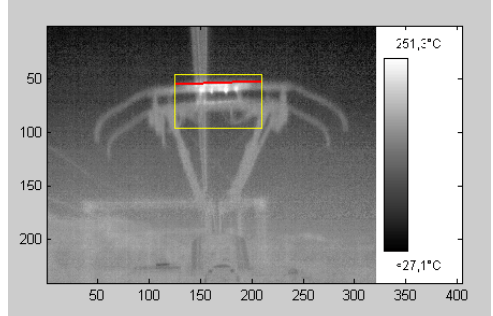

(b)

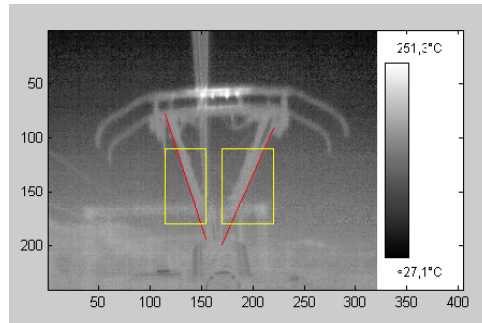

(d)

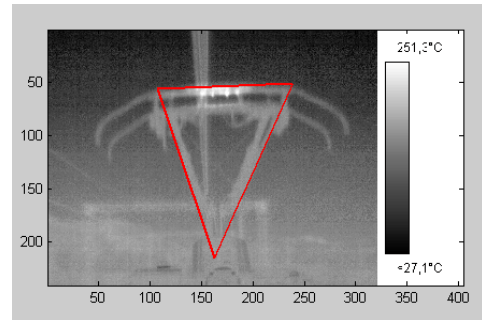

(f)

Figure 2: Application of the Hough transformation. (a) Horizontal detected segments into the reference box of the strip. (b) Upper edge of the nearest strip. (c) Detected segments into the reference boxes of the lateral supports. (d) Detected lateral supports. (e) Detected line by Hough transformation. (f) Reference shape.

one represents the contact line for monitoring (fig. 2.b). Reference box allows following pantograph displacements along a vertical direction. For a correct analysis also the horizontal displacement has to be measured.

Lateral displacements can be then esteemed from an analysis of the two lateral arms sustaining the pantograph heads (fig. 2.c). The two segments representing the external profile of these lateral supports can be identified by repeating the Hough analysis inside two new reference boxes, as shown in (fig. 2.d). The three reference boxes can be joined together for localising a triangle as the reference shape representing the pantograph, as shown in fig. 2.e and fig. 2.f Upper side of the triangle is representing the line useful for monitoring the 
contact with the catenary wires. Note that the representation of the pantograph head with a straight line is only an approximation of the real scenario. Nevertheless the maximum error accumulates in the lateral regions of the segment, where the pantograph head is not rectilinear: fortunately in such region an analysis of the contact is less relevant, since the local temperature is lower than in the middle zone of the segment.

\subsection{Identification of the contact between pantograph and contact wire}

After the identification of the pantograph structure, the following step was devoted to a searching of the contact zone between pantograph and the overhead wire for monitoring the temperature and for checking a correct positioning of the contact wire in terms of its zigzagged movement, relative to the centre line of the track. In the case of the image sequences recorded along the railways from Florence to Rome, it can be observed that there are many straight lines, between them because two overhead wires (fig. 3.a) are in close proximity to the contact region.

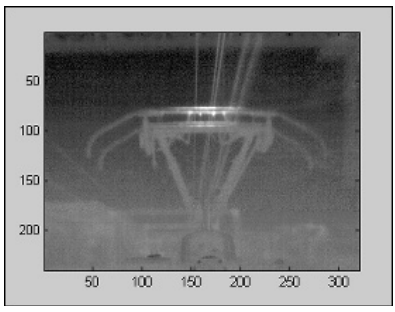

(a)

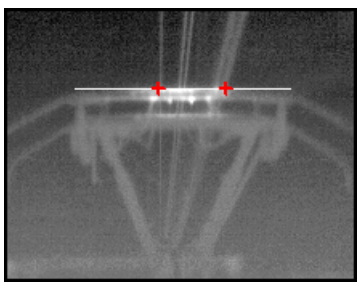

(c)

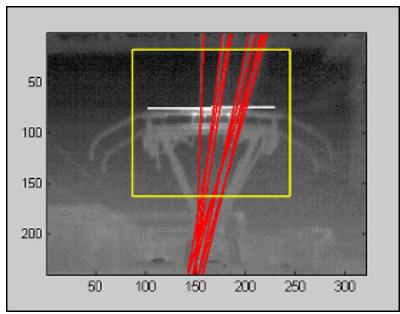

(b)

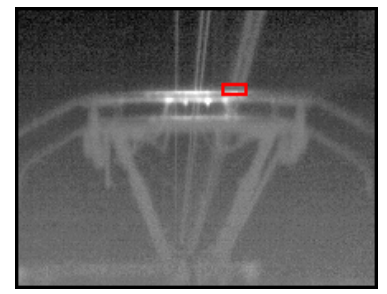

(d)

Figure 3: Identification of the contact between pantograph and overhead contact wire. (a) Image under examination (b) Detected straight lines representing catenary wires. (c) Detected boundaries (d) Identified contact region.

It must be highlighted that the contact points are not corresponding to the hottest points of the strip, usually in the middle of the contact strip. Hough transform can be applied again to detect overhead wires, as shown in fig. 3.b 
many straight lines are detected. A selection of the correct contact wires was performed, based on the two following rules: contact wires are always adjacent and at the exterior of the set of the identified lines and contact wires are thicker than non-contact ones.

The contact points are uniquely located via the following steps:

1) computing the intersection between the upper side of the triangle (pantograph head) and the two straight lines exterior to the pencil of lines (fig. 3.c);

2) analysing the colour distribution of the pixels inside the image, near the two points selected at step 1 . The thickest line of the two is selected, for detecting the contact zone, whose extension is predefined by a fixed number of pixels in the direction of the middle of the pantograph head (fig. 3.d).

\subsection{Passages under the portal structures of the railways}

In the case of a railway line with portal structures (e.g. the railway line connecting Florence to Rome) a similar method can be employed for detecting the poles. The horizontal structure is the easiest element to be recognized: it is the wider structure and it appears even if the poles are not included inside the image, as shown in fig. 4.a.

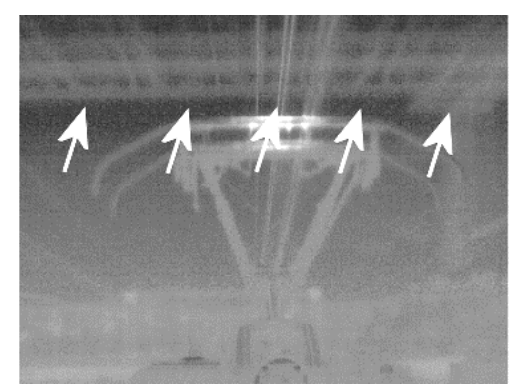

(a)

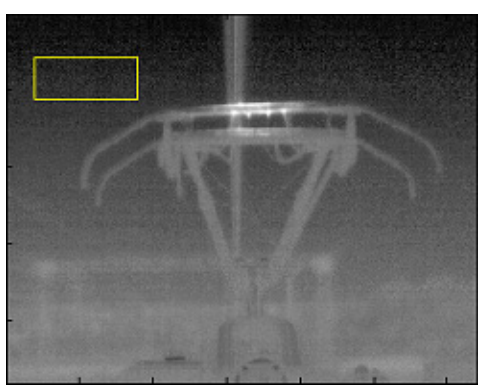

(b)

Figure 4: (a) Horizontal bridge sustaining catenary wires. (b) Observation box for counting the passages of portals.

Therefore counting passages of horizontal portals constitutes a safe method for localising the train position along the line: it can be performed using an observation box positioned in a suitable region of the image. An easy solution is to locate this box in a region where only the horizontal portals are passing, e.g., in the upper left side of each frame (fig 4.b). The Hough transform for straight lines recognizing can be applied inside the observation box and a reliable criterion of portal detection may be based on an accurate analysis of the number of identified lines. Its maximum value is very high in case of passages of portal structures: a threshold value is therefore useful for discriminating noisy images, or single straight lines passages. 


\section{Experimental results}

A preliminary laboratory test was carried out in order to yield the emissivity of the pantograph strip. In Italian Railways ( $3 \mathrm{kV}$ d.c.) the contact material strip is copper (or copper based alloys). By using the IR camera software, the average emissivity of the strip was determined to be 0.2 . The measurement equipment was then installed on board of an ETR500, a high-speed train of Italian Railways. IR-camera (produced by the Flir Systems) was located on the top deck of the locomotive, in front of the pantograph. The block diagram of fig. 5 illustrates the measurement chain. Trial runs have been carried out travelling along the railway line connecting Florence to Rome and vice versa.

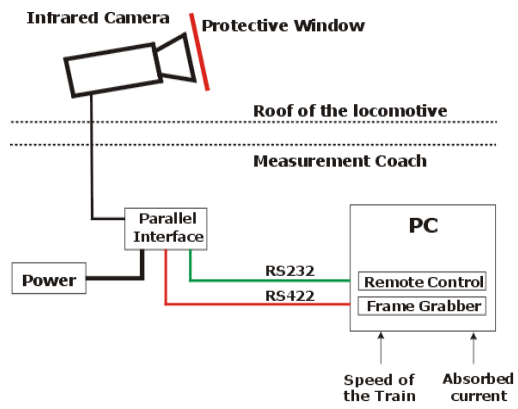

Figure 5: Measurement chain.

\subsection{Portal structure detection an positioning of the overhead contact line}

In fig. 6.a and 6.b two plots are shown, the first one consider a portal structure detection, the second one reports the position of the contact point with respect to the centre of the pantograph head (a positive value means the contact point is located on the right w.r.t. the median position). They are referred to an analysis of 200 frames. Plots are functions of a position relative to the first frame analysed. Train speed was $200 \mathrm{~km} / \mathrm{h}$.

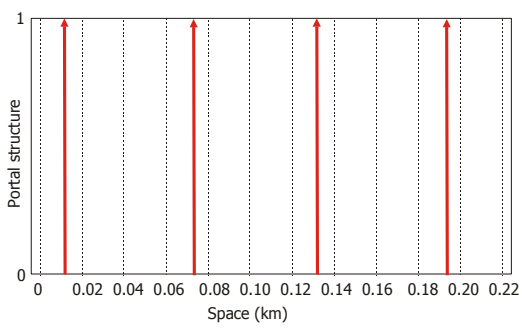

(a)

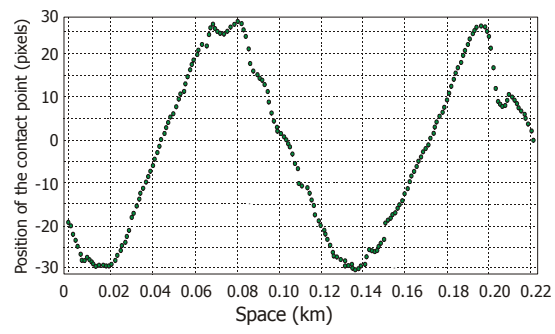

(b)

Figure 6: (a) Positioning of structural portal. (b) Contact point position respect to the centre of the pantograph head. 


\subsection{Example of break arc detection}

Consider now 100 consecutive frames with a break arc corresponding to frame 50, shown in fig.7.a. The observation box is representative of the contact point. The observation box is representative of the contact point.

Temperature profile along the strip surface for frame 50 is shown in fig. 7.b. Figure 8 shows the infrared map of the strip along the railway line connecting Florence to Rome between $\mathrm{km} 60$ and $\mathrm{km} \mathrm{63.} \mathrm{Temperature} \mathrm{peaks} \mathrm{are} \mathrm{correlated}$ with arcing occurrences. This elaboration has been obtained by processing 2700 frame of the video sequence with a computation time of $540 \mathrm{~s}$ (using an AMD $\mathbb{R}$ Athlon processor at $1.2 \mathrm{GHz}$ ).

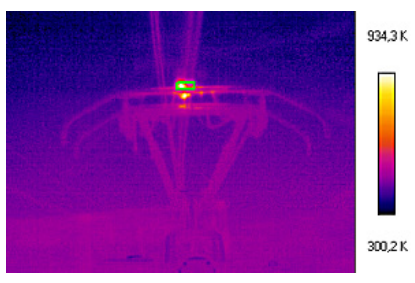

(a)

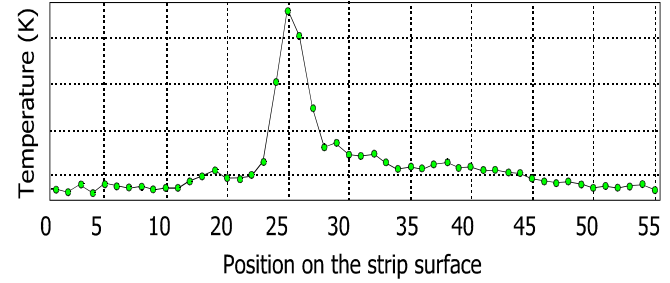

(b)

Figure 7: (a) Frame 50 with break arc detection. (b) Temperature profile on the strip surface.

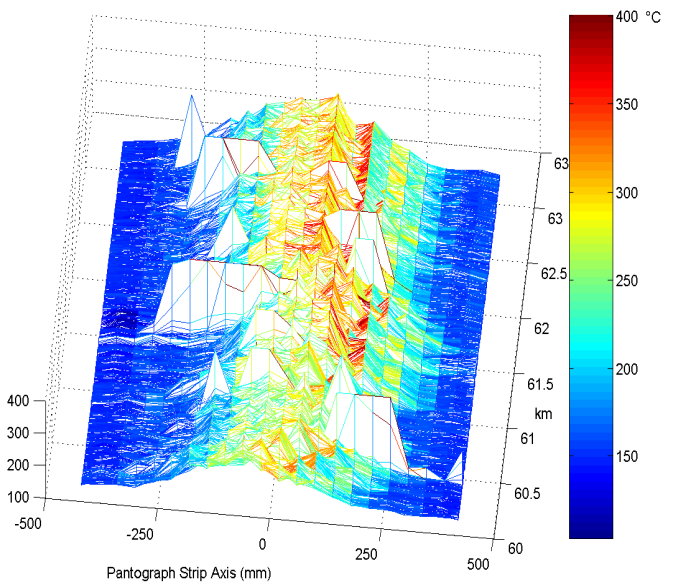

Figure 8: $\quad$ Infrared map of pantograph strip along the railways.

\section{Conclusions}

The use of an infrared camera for monitoring the pantograph-catenary status has been proposed and experimentally tested. The temperature profile of the pantograph strip was detected on infrared images by using the Hough 
transformation. An analysis of the stored images could help maintenance operations, revealing, for example an irregular positioning of the line. New typologies of pantographs, new materials for collector strips, and catenary layout can be thermically analyzed.

\section{Acknowledgement}

The financial support of the Italian Ministry of University and Research - MIUR (Project on Innovative Controls in High-Speed Transport Systems) is gratefully acknowledged.

\section{References}

[1] Bruno, O., Landi, A., Papi, M. and Sani, L. Phototube sensor for monitoring the quality of current collection on overhead electrified railways. In Proc. IMECHE, Part F: Journal of Rail and Rapid Transit, vol.215, n. 3, pp. 231-241, 2001.

[2] Barmada, S., Landi, A., Papi, M. and Sani, L., Wavelet multi-resolution analysis for monitoring the occurrence of arcing on overhead electrified railways, In Proc. IMECHE, Part F: Journal of Rail and Rapid Transit, vol. 217, n 3, pp. 177-187, 2003.

[3] Balestrino, A., Bruno, O., Landi, A., Masini, P., Mingozzi, E., Papi, M. and Sani, L. Infrared camera for monitoring pantograph-catenary interactions. In Proc. of World Conference of Railway Research 2003, Edinburgh (UK), pp. 1-7, 2003.

[4] Runciman, H.M. Thermal imaging, in Measurement, Instrumentation, and Sensors Handbook CRCnetBase 1999, J.G. Webster Editor, CRC Press, 2000 .

[5] Hough, P. Method and means for recognizing complex patterns, in U.S. Patent 3069654, 1962.

[6] Deans, S, R. Hough Transform from the Radon Transform, in IEEE Transactions on Pattern Analysis and Machine Intelligence Vol. PAMI-3, No 2, pp.185-188, (Mar.1981).

[7] Canny, J. A Computational Approach to Edge Detection, in IEEE Transactions on Pattern Analysis and Machine Intelligence Vol. PAMI-8, No 6, pp.679-698, (Nov.1986). 